\title{
Die pyloruserhaltende Whipple-Operation: eine Alternative oder heutiger Standard beim Pankreaskarzinom?
}

\author{
Ch. Sadowski, W. Uhl, H. U. Baer, Ch. Seiler und M. W. Büchler
}

Schliusselwörter: Pankreaskarzinom - partielle Duodenopankreatektomie - Whipple-Operation - pyloruserhaltende Whipple-OperationLebensqualität - Überleben.

Key-words: Carcinoma of the pancreas - partial duodenopancreatectomy - Whipple procedure - pylorus-preserving duodenopancreatectomy - life quality - survival.

Zusammenfassung: Grundlagen: Während die pyloruserhaltende Whipple-Operation eine gute Alternative zur klassischen Operation bei periampullären Tumoren darstellt, ist das Verfahren beim Pankreaskopfkarzinom umstritten

Methodik: Wir haben die Daten von 70 Patienten mit Pankreaskopfkarzinom prospektiv im Hinblick auf die Vorteile des operativen Vorgehens und in bezug auf Lebensqualität und Überlebenszeit untersucht.

Ergebnisse: Bei 44 Patienten wurde eine pyloruserhaltende Resektion durchgeführt, bei 26 Fällen eine klassische Whipple-Operation (WO) mit Lymphadenektomie. Es gab bezüglich Alter, Geschlecht und Tumorstadium keine Unterschiede in den beiden Patientengruppen. Die Mittelwerte für die Operationszeit, Blutverlust und Hospitalisationsdauer betrugen bei der pyloruserhaltenden WO $382 \mathrm{~min}, 1125 \mathrm{ml}$ und 18,3 Tage und bei der klassischen WO $460 \mathrm{~min}(\mathrm{p}<0,05), 1650 \mathrm{ml}(\mathrm{p}<0,05)$ und 22 Tage. Die mediane Überprüfzeit betrug 17 Monate (Range 2 bis 28 Monate). In den ersten 3 Monaten postoperativ war der Gewichtsverlauf bei den Patienten mit pyloruserhaltender WO deutlich besser. Die mediane Überlebenszeit war nicht signifikant verschieden mit 12,2 Monaten für die Patienten mit pyloruserhaltender WO und 12,9 Monaten bei den Patienten mit klassischer WO.

Schlußfolgerungen: Bei Patienten mit Pankreaskarzinom war die Lebensqualität und Gewichtszunahme postoperativ besser bei solchen mit pyloruserhaltender partieller Duodenopankreatektomie im Vergleich zur klassischen WO. Aufgrund dieser Ergebnisse, der intraoperativen Vorteile und bei gleicher Überlebenszeit sollte dieses Operationsverfahren beim Pankreaskarzinom, wenn immer möglich, bevorzugt werden.

(Acta Chir. Austriaca 1997;29:271-274)

Pylorus Preserving Whipple Procedure: An Alternative or Todlay's Standard Procednre in Patients with Pancreatic Carcinoma?

Summary: Background: While the pylorus-preserving Whipple procedure is a good alternative procedure to the classical Whipple operation in patients with periampullary tumors, in patients with pancreatic cancer this technique is controversially discussed in the literature.

Methods: We analyzed the data of the last 70 consecutively hospitalized patients with carcinoma of the pancreas with regard to the advantages of the operation, life quality and survival time.

Results: In 44 patients a pylorus-preserving procedure was performed, in 26 cases a classical Whipple operation. There were no

Korrespondenzanschrift: Prof. Dr. MI. W. Büchler, Klinik für Viszerale und Transplantationschirurgie, Universität Bern, (Inselspital), Murtenstraße 35, CH-3010 Bern, Schweiz. differences concerning age, sex, and tumor stage in the two patient groups. The mean operation time, blood loss and days the patients were hospitalized were for the group undergoing pylorus-preserving Whipple procedure $383 \mathrm{~min}, 1125 \mathrm{ml}$ and 18.3 days and in the group with classical Whipple procedure $460 \mathrm{~min}$ $(\mathrm{p}<0.05), 1650 \mathrm{ml}(\mathrm{p}<0.05)$ and 22 days, respectively. The median observation time was 17 months (range 2 to 28 ). In the following, we found a better weight course postoperatively for the pylorus-preserving Whipple procedure group. The median survival time was not significantly different (12.2 months for the pylorus-preserving and 12.9 months for the classical Whipple procedure).

Conclusions: For patients with pancreatic cancer life quality and weight gain was better in those who had undergone the pyloruspreserving procedure in comparison with the patients with classical Whipple operation. We conclude, that because of these results and the intraoperative advantages and the same length of survival for patients with carcinoma of the pancreas this operation technique should be prefered.

\section{Einfuihrang}

Kausch und später Whipple haben mit der partiellen Duodenopankreatektomie mit Resektion des Magens einen Meilenstein in der Pankreaschirurgie gelegt (18). Jahrzehnte wurde diese Operation in verschiedenen Modifikationen zur Resektion des Pankreaskopfkarzinoms angewendet. 1942 hat Watson (33) erstmals die Möglichkeit des Magenerhaltes, inklusive Pylorus und proximalen Duodenums bei Papillentumoren im Rahmen der Duodenopankreatektomie beschrieben. Er erhoffte sich davon Vorteile, wie die Vereinfachung der Operationstechnik und eine bessere Gastrointestinalfunktion mit günstigerem postoperativem $\mathrm{Ge}$ wichtsverlauf. Dieses operative Konzept ging in der Folge in Vergessenheit, bis 1978 Traverso und Longmire (28) die pyloruserhaltende Duodenopankreatektomie ,,wiederentdeckten“".

In den letzten Jahren gehen die Meinungen in bezug auf die Erhaltung des Pylorus beim Pankreaskopfkarzinom auseinander. Von einigen Autoren $(26,18$ ) wird eine ungenügende Radikalität beim Malignom befürchtet, andere sind der Ansicht, daß die Radikalität und damit die Prognose nicht von der Magenresektion, sondern von der Lymphknotendissektion $(1,25,11,13)$ abhängig ist.

Die vorliegende Arbeit vergleicht die intraoperativen Eckdaten, Morbidität und Letalität sowie die postoperative Lebensqualität unserer Patienten mit Pankreaskopfkarzinom der letzten 3 Jahre, bei denen eine pyloruserhaltende oder eine klassische Whipple-Operation (wO) durchgeführt wurde und stellt diese den Ergebnissen der Literatur gegeniber.

\section{Patientern und Methodik}

Von November 1993 bis August 1996 wurden 130 Patienten mit einem Pankreaskarzinom am Universitätsspital Bern operativ behandelt. Bei 41 Patienten mit fortgeschrittenem Tumorleiden (Stadium IV nach UICC) wurde keine Resektion durchgeführt, bei 19 weiteren Fällen wurde eine Linksresektion oder totale Pankreatektomie durchgeführt. Pankreaskopfresektionen wurden bei insgesamt 70 Patienten mit Pankreasneoplasma vorgenommen. Bei 44 Patienten wurde ein pyloruserhaltender, bei $26 \mathrm{~Pa}$ tienten eine klassische WO durchgeführt (Tab. 1). Von diesen 70 
Tab. 1. Beschreibung der Patienten-Kollektive mit pyloruserhaltender und klassischer Whipple-Operation.

\begin{tabular}{|l|c|c|}
\hline & $\begin{array}{c}\text { pyloruserhaltende } \\
\text { Whipple-Operation } \\
(\mathbf{n = 4 4 )}\end{array}$ & $\begin{array}{c}\text { klassische } \\
\text { Whipple-Operation } \\
(\mathbf{n = 2 6})\end{array}$ \\
\hline Geschlecht $\mathrm{m} / \mathrm{w}$ & $29 / 15$ & $15 / 11$ \\
\hline Alter (Jahre) & $65,2(31$ bis 78$)$ & $66,0(28$ bis 84$)$ \\
\hline UICC I & $4(9 \%)$ & $3(11 \%)$ \\
\hline UICC II & $16(36 \%)$ & $11(42 \%)$ \\
\hline UICC III & $24(55 \%)$ & $12(47 \%)$ \\
\hline UICC IV & - & - \\
\hline
\end{tabular}

Patienten mit WO waren 44 männlich, 26 weiblich, das Durchschnittsalter betrug 65,3 Jahre (Range 28 bis 84 Jahre). Nach der UICC-Klassifikation lag bei 7 Patienten ein Stadium I, bei 27 ein Stadium II, und bei 36 ein Tumorstadium III vor.

Bei beiden Operationen wurde für die Rekonstruktion eine Jejunumschlinge verwendet, welche nach dem Roux-Y-Prinzip retrokolisch hochgezogen wurde. Es wurde entweder eine End-zuSeit- oder End-zu-End-Pankreatikojejunostomie angelegt. Die Choledocho- oder Hepatikojejunostomie wurde in die gleiche Schlinge anastomosiert. Perioperativ erhielten alle Patienten eine Antibiotikaprophylaxe mit Piperacillin und Metronidazol sowie eine exokrine Sekretionshemmung mit Octreotid $(3 \times 100 \mu \mathrm{g})$ über 7 Tage $(4,10,17,21)$.

In den beiden Gruppen wurden die Blutverluste, Operationsdauer, Dauer des Belassens der Magensonde und die Hospitalisationsdauer ausgewertet. Als Klinikletalität wurde das Ereignis des Todes während der Hospitalisation definiert.

Alle Patienten wurden in regelmäßigen Abständen (12-WochenIntervallen) nachkontrolliert. Die mediane postoperative Kontrollzeit betrug 17 Monate (Range 2 bis 28 Monate). Zur Berechnung signifikanter Unterschiede wurde der Chi-Square-Test oder Wilcoxon-Test für unverbundene Stichproben verwendet mit einem Signifikanzniveau von $\mathrm{p}<0,05$.

\section{Ergebnisse}

Die beiden Gruppen zeigten vergleichbare Tumorstadien: Es befanden sich 9 und $11 \%$ im Stadium I, 36 und $42 \%$ im Stadium II und 55 und $47 \%$ im Stadium III bei den Patienten mit pyloruserhaltender bzw. klassischer WO (Tab. 1).

Die Dauer der Operation bei 44 Patienten mit pyloruserhaltender WO betrug im Mittel 382 min (Range 300 bis $525 \mathrm{~min}$ ) mit einem medianen Blutverlust von $1125 \mathrm{ml}(600 \mathrm{bis} 3000 \mathrm{ml})$, bei den 26 Patienten mit klassischer WO ergaben sich im Vergleich $460 \mathrm{~min}$ (Range $300 \mathrm{bis} 635 \mathrm{~min}$ ) und $1650 \mathrm{ml}$ (600 bis $3500 \mathrm{ml}$ ) (Tab. 2). Die Unterschiede für Operationsdauer, Blutverlust und Notwendigkeit für Bluttransfusionen waren signifikant besser bei pyloruserhaltender WO $(\mathrm{p}<0,05)$.

Der Vergleich der postoperativen Komplikationen zeigte ein Vorwiegen der Magenentleerungsstörungen bei den Patienten mit klassischer WO $(p<0,05)$ (Tab. 3). Die postoperative Liegedauer der Magensonde betrug 3,4 Tage (Range 1 bis 30 Tage) für die pyloruserhaltende WO und 12,4 Tage (Range 1 bis 59 Tage) bei den Patienten mit klassischer WO. Total war die postoperative Morbidität in beiden Gruppen aber gleich (30\%). Die Montalität betrug $0 \%$ in beiden Gruppen. Bezïglich der Hospitalisationsdauer fand sich kein signifikanter Unterschied: Diese betrug bei pyloruserhalten-

Tab. 2. Operationsdaten bei pyloruserhaltender und klassischer Whipple-Operation.

\begin{tabular}{|l|c|c|c|}
\hline & $\begin{array}{c}\text { pyloruserhaltende } \\
\text { Whipple-Operation } \\
(\mathbf{n}=44)\end{array}$ & $\begin{array}{c}\text { klassische } \\
\text { Whipple-Operation } \\
(\mathbf{n}=\mathbf{2 6})\end{array}$ & Signifikanz \\
\hline Operationszeit (min) & $382(300$ bis 525$)$ & $460(300$ bis 635$)$ & $\mathrm{p}<0.05$ \\
\hline Blutverlust $(\mathrm{ml})$ & $1125(600$ bis 3000$)$ & $1650(600$ bis 3500$)$ & $\mathrm{p}<0.05$ \\
\hline $\begin{array}{l}\text { EK intraoperativ } \\
\text { (Mittelwert) }\end{array}$ & $1,2(0$ bis 4$)$ & $1,8(0$ bis 7$)$ & $\mathrm{p}<0,05$ \\
\hline
\end{tabular}

der WO 18,3 Tage (Range 8 bis 34 Tage) gegenüber 22 Tagen ( 9 bis 45 Tage) bei klassischer WO.

Der Gewichtsverlauf während der ersten 3 Monaten postoperativ war bei den Patienten mit pyloruserhaltender WO deutlich günstiger (Tab. 4). Während bei klassischer WO 77\% der Patienten über 5 bis $10 \mathrm{~kg}$ im Vergleich zum präoperativen Ausgangsgewicht abnahmen, lag der Prozentanteil bei pyloruserhaltender WO bei $47 \%$. $12 \%$ der Patienten hatten sogar wieder eine Gewichtszunahme mit bis zu $5 \mathrm{~kg}$ in diesem Kollektiv verglichen mit $0 \%$ beim klassischen Vorgehen.

Lokalrezidive traten im Median nach 9 Monaten (Range 3 bis 26 Monate) auf, darin bestand kein Unterschied in den beiden Gruppen. Ebenso war die mediane Überlebenszeit nicht verschieden mit 12,2 Monaten bei pyloruserhaltender WO (Range 3 bis 27 Monate) und 12,9 Monate bei klassischer WO (Range 2 bis 28 Monate). In beiden Gruppen war die 1-Jahres-Überlebenszeit mit 60 und $54 \%$ nicht unterschiedlich (Tab. 5).

\section{Diskussion}

Jahrzehnte war bei Pankreaskopfkarzinomen die klassische WO die Standardoperation. Um die postoperative Morbidität und Letalität zu senken, haben Traverso und Longmire (28) 1978 bei Patienten mit chronischer Pankreatitis und malignen sowie benignen Papillentumoren die Methode der Pyloruserhaltung bei Whipple ,wiedereingeführt", die von Watson erstmals 30 Jahre zuvor beschrieben wurde. Man erwartete sich davon weniger Dumping-Syndrome, eine bessere Gastrointestinalfunktion, weniger Anastomosenulzera, eine bessere postoperative Gewichtszunahme und damit eine Verbesserung der Lebensqualität.

In jüngster Zeit wird diese Operationsmethode zunehmend beim Pankreaskarzinom eingesetzt, aber kontrovers diskutiert. Noch 1982 hat Moosa (18) publiziert, daß die pyloruserhaltende WO nicht beim Karzinom durchgeführt werden soll. Andererseits haben Newmann et al. (20) festgestellt, daß die Resektionsränder bei der Duodenopankreatektomie zum Retroperitoneum und den Gefäßen viel näher am Tumor liegen als zum Duodenum und Antrum. Sie schlußfolgerten daraus, daß Schnellschnittentnahmen während der Operation geniigen, um im Bereich des Duodenumresektionsrandes sicher über die Radikalität zu sein.

Wir führen eine möglichst radikale Resektionstechnik bei Patienten mit Pankreaskopfkarzinom durch. Nach Eröffnung des Abdomens durch eine mediane Laparotomie und Kocher-Manöver wird der Pankreaskopf in bezug auf Resektabilität evaluiert. Eine kritische Frage ist, ob eine komplexe Gefäßinfiltration des Truncus coeliacus oder der Mesenterialgefäße vorliegt oder nicht. Eine Tumorinfiltration der V. portae wird nicht als eine Kontraindikation für eine Resektion angesehen. Eine ausgedehnte Lymphknotendissektion gehört heute zum chirurgischen Standard der Operation. Bei der Pankreasanastomose ziehen wir eine End-zu-End-Pankreatikojejunostomie wenn immer möglich vor. Ist der Ductus pancreaticus jedoch eng, ist es besser, eine End-zu-Seit-Anastomose durchzuführen.

Wir haben in unserem Patientengut eine kürzere Operationszeit und weniger Blutverlust nach pyloruserhaltender WO gefunden. Postoperative Komplikationen traten in beiden Gruppen bei $30 \%$ der Patienten auf. DaB nur 2 Pankreasfisteln (3\%) aufgetreten sind. sehen wir als Folge der standardisierten Operacionstechnik und der perioperativen exokrinen Sekretionshemmung $(4,10,17,21)$. Die Letalität war in beiden Gruppen $0 \%$. Die häufigste Komplikation nach Pankreatikoduodenektomie ist die Magenentleerungsstörung. Während diese früher in der Literatur $(3,13)$ vorwiegend bei pyloruserhaltender WO beschrieben wurde, haben die neueren prospektiven Studien gezeigt, daß diese Komplikation oft bei klassischer WO auftritt $(11,15$, 24,32 ). In unserer Untersuchung war das Auftreten von Magenentleerungsstörun- 
gen signifikant häufiger bei klassischer WO (19\% vs. 9\%; p < $0,05)$.

Eine intakte neurovaskuläre Versorgung des Pylorus ist gemäß Fernandez et al. (8) für eine gute Magenentleerung von Bedeutung. Daher sollte die A. gastrica dextra erhalten werden, während die rechte Gastroepiploica und Gastroduodenalis ligiert werden können. In unserem Patientenkollektiv sind keine Anastomosenulzera aufgetreten. Klinische Studien haben das Auftreten von Ulzera bei beiden Operationen erwähnt (12). Traverso et al. (28) haben experimentell mit Gastrinstimulationsmessungen gezeigt, daß keine physiologische Grundlage für die Vermutung besteht, daß Ulzera nach pyloruserhaltender WO häufiger auftreten sollen als nach klassischer WO.

Nach einer klassischen WO können die meisten Patienten ihr präoperatives Gewicht nicht wieder erreichen. Es wurde gezeigt (30), daß durch die Erhaltung des Magens neben der Reservoirfunktion eine physiologische Regulation der gastrointestinalen Hormone erreicht wird. Das führt zur Vermeidung von enterogastrischem Reflux, normalen Vitaminspiegeln und besserem Gewichtsverlauf postoperativ. In unserem Patientenkollektiv war der Gewichtsverlauf übereinstimmend mit den Literaturdaten bei pyloruserhaltender WO deutlich besser.

Verschiedene Autoren bezweifeln vor allem die Radikalität $(18,26)$ der pyloruserhaltenden WO bei malignen Tumoren des Pankreaskopfes, andere berichten über eine verlängerte Hospitalisation als Resultat der in dieser Gruppe vermehrt auftretenden Magenentleerungsstörung $(3,21)$ mit einer postoperativ verlängerten Rekonvaleszenzphase und Einschränkung der Lebensqualität. Grace et al. (I1) berichteten anhand der eigenen Patienten 1990, daß die pyloruserhaltende WO eine radikale und sichere Operation ist mit vergleichbarer Morbidität und Letalität bei gutund bösartigen periampullären Tumoren.

1990 sind die ersten Arbeiten erschienen, welche Zahlen in bezug auf das Überleben nach pyloruserhaltender und klassischer WO angeben $(1,11,14,25,26,30)$. Roder et al. (26) sind der Meinung, daß die klassische WO beim Pankreaskarzinom im Stadium III bessere Resultate ergibt als die pyloruserhaltende WO, im Gegensatz dazu fanden sich im Tumorstadium I und II keine Unterschiede. Klinkenbjil et al. (14) hingegen haben bei $44 \mathrm{~Pa}-$ tienten mit klassischer WO und 47 mit pyloruserhaltender WO keine Unterschiede bezüglich Rezidiv (nach 11 Monaten im Durchschnitt, Range 3 bis 38 Monate) und Überlebensraten (1Jahres-Überlebensrate $56 \%$, 2-Jahres-Überlebensrate $38 \%$ ) gefunden. Der Zeitpunkt des Rezidivauftrittes ( 6 Monate in beiden Gruppen, Range 2 bis 27) und die mittlere Überlebenszeit (12,2 und 12,9 Monate, Range 2 bis 28) in unserem Patientengut zeigte in beiden Gruppen ebenfalls keinen Unterschied, ebenso war die 1-Jahres-Überlebensrate nicht signifikant verschieden $(60 \%$ bei pyloruserhaltender WO, $54 \%$ bei klassischer WO).

In bezug auf das Überleben nach der Operation finden wir keinen Nachteil durch die Erhaltung des Magens und Pylorus, denn die Lymphknotenstationen können auch bei dieser Operation bis paraaortal links entfernt werden, was im Hinblick auf die Radikalität von größerer Bedeutung ist (15) als die ausgedehnte Organresektion inklusive Magenteilentfernung.

Bezüglich Überlebenszeit wird in der Literatur mehrheitlich (1, 7) erwähnt, daß ein positives Lymphknotenstadium ein wichtiger prognostischer Faktor ist. Akao et al. (1) fanden bei 90 Pankreaskarzinomen positive Lymphknoten perigastrisch in $77 \%, 51 \%$ hinter dem Pankreaskopf, 39\% paraaortal und $23 \%$ im Bereiche hinter der A. mesenterica superior. Daher schlagen sie zur Prognoseverbesserung eine ausgedehnte Lymphknotendissektion vor. Eine der Schwierigkeiten festzustellen, ob eine radikalere Operation bessere Resultate bringt, besteht aber darin, daß einerseits Chirurgen und andererseits Pathologen sich hinsichtlich auf das Lymphknotenstadium oft zu wenig klar äußern (25).

Zusammenfassend sind wir der Meinung, daß bei Patienten mit Pankreaskoptkarzinom ein möglichst organsparender Eingriff mit Erhalt von Magen und Pylorus bei maximaler Radikalität (Lymphknotendissektion) durchgeführt werden soll, um eine gute Lebensqualität für die verbleibende Zeit nach der Operation
Tab. 3. Postoperative Morbidität, Hospitalisationsdauer und Letalität bei pyloruserhaltender und klassischer Whipple-Operation.

\begin{tabular}{|l|c|c|}
\hline Morbidität & $\begin{array}{c}\text { pyloruserhaltende } \\
\text { Whipple-Operation } \\
(\mathbf{n = 4 4 )}\end{array}$ & $\begin{array}{c}\text { klassische } \\
\text { Whipple-Operation } \\
(\mathbf{n = 2 6 )}\end{array}$ \\
\hline Pankreasfistel & 2 & 0 \\
\hline Pncumonie & 1 & 1 \\
\hline Wundinfekt & 2 & 0 \\
\hline Urininfekt & 2 & 1 \\
\hline Herzkreislauf & 1 & 1 \\
\hline LeberabszeB & 1 & 0 \\
\hline Magenentleerungsstörung & $4(9 \%)$ & $5(19 \%)$ \\
\hline Total & $\mathbf{1 3 ( 3 0 \% )}$ & $\mathbf{8}(\mathbf{3 0 \%})$ \\
\hline Letalität & 0 & 0 \\
\hline Hospitalisationsdauer (Tage) & $18,3(8$ bis 34) & $22(9$ bis 45$)$ \\
\hline
\end{tabular}

Tab. 4. Postoperativer Gewichtsverlauf nach 3 Monaten bei pyloruserhaltender und klassischer Whipple-Operation im Vergleich zum präoperativen Gewicht.

\begin{tabular}{|l|c|c|}
\hline & $\begin{array}{c}\text { pyloruserhaltende } \\
\text { Whipple-Operation } \\
(\mathbf{n}=44)\end{array}$ & $\begin{array}{c}\text { klassische Whipple- } \\
\text { Operation } \\
(\mathbf{n}=26)\end{array}$ \\
\hline-5 bis $-10 \mathrm{~kg}$ & $21(47 \%)$ & $20(77 \%)$ \\
\hline 0 bis $-5 \mathrm{~kg}$ & $18(41 \%)$ & $6(23 \%)$ \\
\hline 0 bis $+5 \mathrm{~kg}$ & $5(12 \%)$ & 0 \\
\hline
\end{tabular}

Tab. 5. Zeitpunkt des Alffretens eines Lokalrezidivs und Überlebenszeiten nach pyloruserhaltender und klassischer Whipple-Operation.

\begin{tabular}{|l|c|c|}
\hline & $\begin{array}{c}\text { pyloruserhaltende } \\
\text { Whipple-Operation } \\
(\mathrm{n}=44)\end{array}$ & $\begin{array}{c}\text { klassische Whipple- } \\
\text { Operation } \\
(\mathrm{m}=26)\end{array}$ \\
\hline Auftritt Lokalrezidiv & $9(3$ bis 27$)$ & $9(2$ bis 26$)$ \\
\hline $\begin{array}{l}\text { mediane Überlebenszeit } \\
\text { (Monate) }\end{array}$ & $12,2(3$ bis 27$)$ & $12,9(2$ bis 28$)$ \\
\hline 1-Jahres-Überlebenszeit & $60 \%$ & $54 \%$ \\
\hline
\end{tabular}

zu erzielen. Zusätzlich führt das Verfahren der pyloruserhaltenden WO zu einer Einsparung von Operationszeit, Blutverbrauch und zu einer kürzeren Hospitalisationsdauer und zu besserer postoperativer Magendarmfunktion und ebensolchem Gewichtsverlauf.

\section{Literatur}

(1) Akuo A. Harada T. Nomani T. Kaneko H. Murakami S: Lymph node metastases in carcinoma of the pancreas. J Surg 1995:82:399-402.

(2) Bittner R, Roscher R. Safi F: Der Einfluss von Tumorgröße und Lymphknotenstatus auf die Prognose des Pankreaskarzinoms. Chirurg 1989;60:240-245.

(3) Braasch JW. Rossi RL. Diezel DJ: Pyloric and gastric preserving pancreatic resection. Ann Surg 1986:4:411-418.

(4) Büchler NW. Friess H Klempa l, et al: Role of octreotide in the prevention of postoperative complications following pancreatic resection. Am I Surg 1992:163: 125-131.

(5) Büchler M. Ebert M. Beger HG: Grenzen chirurgischen Handelns beim Pankreaskarzinom. Langenbecks. Arch Chir Suppl (Kongressbericht 1993).

(6) Cameron JL. Crist DW. Sizmann JV: Factors influencing survival after pancreaticoduodenectomy for pancreatic cancer. Am J Surg 1991:161:120-125.

(7) Cubilla AL, Fortner J. Fizgerald PJ: Lymph node involvement in carcinoma of the head of the pancreas area. Cancer 1978:41:880-887.

(8) Fernandez L. Gonzalez S, Saenz A: Ischemic injury to the antropyloric segment possible mechanism for gastric stasis following pyloruspreserving pancreaticoduodenectomy. Dig Surg 1999 +:1 1:397-401.

(9) Fink AS. DeSouza LR. Vayer EA: Long term evaluation of pylorus preservation (9) Fink AS. DeSouza LR. Mayer EA. Long term evaluation of py
during pancraticoduodenectomy. World J Surg 1988:12:663-670.

(10) Friess H. Beger HG. Sulkowski U, et al: Randomized controlled multicentre study of the prevention of complications by octreotide in patients undergoing surgery for chronic pancretitis. Br J Surg 1995;82:1270-1273.

(1) Grace PA. Pitt HA. Longmire WP: Pylorus preserving pancreaticoduodenectomy: an oversiew. Br J Surg 1990:70:968-974.

(12) Grant CS. Van Heerden JA: Anastomotic ulceration following subtotal and total pancreatectomy. Ann Surg 1979:190:1-5. 
(13) Itani K, Colemann RE. Meyers WC, Akwardi OE: Pylorus-preserving Pancreaticoduodenectomy. Ann Surg 1986;204:655-664

(14) Klinkenbijl JH, Schelling GP, Hop WC, et al: The advantages of pyloruspreserving pancreatoduodenectomy in malignant disease of the pancreas and periampullary region. Ann Surg 1992:216:142-146.

(15) Kozuschek Wreith HB. Haarmann W: The role of pyloruspreserving duodenopancreatic head resection. In Beger HG, Büchler MW, Malfertheiner P (eds): Standards in pancreatic surgery. Berlin, Springer 1993, p 114.

(16) Livingston EH, Welton ML, Reber HA: The United States experience with surgery of the pancreatic cancer. Int J Pancreatol 1991;9:153.

(17) Montorsi M, Zago M, Mosca F, et al: Efficacy of octreotide in the prevention of pancreatic fistula after elective pancreatic resections. Surgery 1995;117:26-31

(18) Moossa AR: Pancreatic cancer: Approach to diagnosis, selection for surgery and choice of of operation. Cancer 1982;50:2699-2698

(19) National cancer institute: annual cancer statistics review 1973-1988.

(20) Newmann KD, Braasch JW, Rossi RL, et al: Pyloric and gastric preservation with pancreaticoduodenectomy. Am J Surg 1985;145:152.

(21) Pederzoli P, Bassi C, Falconi M, et al: Efficacy of octreotide in the prevention of complications in elective surgery. Br J Surg 1994:81:265-269.

(22) Pitt HA: Curative treatment for pancreatic neoplasm. Surg Clin NA (22) Pitt HA:

(23) Pitt HA, Grace PA: Pyloruspreserving resection of the pancreas. Baill Clin Gastroent 1990;4:917-929.

(24) Reber HA, Stanley W, Ashley W: Curative treatment for pancreatic neoplasm. Surg Clin NA 1995:75:905-912.
(25) Roder ID, Stein HJ, Huett W: Pyloruspreserving versus standard pancreaticoduodenectomy: an analysis of 110 pancreatic and periampullary carcinomas. $\mathrm{Br}$ Surg 1992:9:152-155.

(26) Sadowski CE, Uhl W, Baer HU, Büchler MW: Delayed gastric emptying after classic and pyloruspreserving Whipple procedure: a prospective study. Dig Surg 1997; 14:159-164.

(27) Takada T: Pyloruspreserving pancreaticoduodenectomy: technique and indications. Hepato Gastroent 1993:40:422-425.

(28) Traverso LW, Longmire WP: Preservation of the pylorus in pancreaticoduodenectomy. Ann Surg 1980;192:306-310.

(29) Trede MA, Schwall G, Saeger HD: Survival after pancreatoduodenectomy. Ann Surg 1990;211:447-458.

(30) Tsao JI, Rossi RL, Lowell JA: Pyloruspreserving pancreaticoduodenectomy. Arch Surg 1994:129:405-411.

(31) Tsuchiya R. Tsunoda T, Ishida $T$ : Resection for cancer of the pancreas-the Japanese experience. Baill Clin Gastroent 1990;4:931-939.

(32) Warshaw AL, Torchiana DL: Delayed gastric emptying after pylorus-preserving Whipple surgery. Surg Gynecol Obstet $1985 ; 160: 1-4$.

(33) Watson $\mathrm{K}$ : Carcinoma of ampulla of Vater: successful radical resection. $\mathrm{Br} \mathrm{J}$ Surg 1944;31:368-373.

(34) Whipple AO, Parson WB. Mullins CR: Treatment of the carcinoma of the ampulla of Vater. Ann Surg 1935:102:763-769.

(35) Yeo CJ: Management of complications following pancreaticoduodenectomy. $S$ Clin NA 1995;5:913-924.

\section{Internationales Symposium am 12. Dezember 1997 im AKH Wien über die Rolle von G-CSF (Filgrastim, Neupogen) im Rahmen der Infektiologie}

In den letzten Jahren wurde neben der Hebung der Zahl der neutrophilen Granulozyten im peripheren Blut durch G-CSF auch die Funktionsverbesserung ebendieser Zellen intensiv untersucht.

Dabei wurde zunächst in vielen tierexperimentellen Modellen nachgewiesen, daß G-CSF die Funktion normaler Neutrophiler verbessert, und, prophylaktisch gegeben, auch Funktionsstörungen (durch Operation, Trauma, Verbrennungen) reduzieren und die damit verbundene Morbidität und Letalität senken kann.

Der Begriff der ,funktionellen Neutropenie”, die durch G-CSF korrigiert werden kann, beschreibt Zustände, bei denen die bestehenden Neutrophilenspiegel nicht ausreichen, um schwere Infektionen suffizient zu bekämpfen und / oder zu vermeiden.

Nunmehr liegen auch die ersten vielversprechenden klinischen Ergebnisse für einige Indikationen vor.

Ziel dieses Symposiums soll sein, die bisher vorliegenden Daten (Klinik und Labor) zu präsentieren, die ,,funktionelle Neutropenie" zu diskutieren und allen Teilnehmern die Möglichkeit zu bieten, mit den wenigen Experten, die in den bisherigen Entwicklungsprozeß aktiv eingebunden waren, in Diskussion zu treten.

Wir möchten Sie daher namens Prof. Dr. Dr. W. Graninger herzlich zu dieser Veranstaltung einladen.

Richten Sie Ihre Anmeldung bitte an: Mag. R. Weidinger, AMGEN Österreich, Handelskai 388/661, A-1020 Wien, Fax +43/1/72943 57-20, e-mail: richardw@amgen.com

\section{Das Programm:}

\section{International Symposium Infectiology:}

Wellknown Problems - New Solutions?

\section{$\mathbb{G}=\mathrm{CSE}$ (Filgrastim, Neupogen ${ }^{\circledR}$ ) in 'Functional Neutropenia'}

Scientific Organisation:

Universitätsklinik für Innere Medizin I

Chair:

Prof. Dr. Dr. W. Graninger

Verue:

Friday, December 12, 1997, 1.15 p.m.

Lecture Hall 1, Floor 8

AKH Vienna, Austria

\section{PROGRAM}

13.15 Introduction

13.30 G-CSF (filgrastim) as new agent in infectious diseases - the bench results

14.00 Improved antibiotic efficacy through G-CSF (filgrastim) - one possible explanation for its positive results in clinical trials

14.30 G-CSF (Filgrastim) in pneumonia clinical trial results

15.00 Break

15.30 G-CSF (filgrastim) - a new perspective in the treatment of diabetic foot patients?

16.00 G-CSF (filgrastim) - a new player in the perioperative setting: clinical results of esophagectomies

16.30 Summary and closing remarks
W. Graninger, Vienna

T. Hartung, Konstanz

J. Andresen, TO

R. K. Root, Washington

M. Edmonds, London

A. Engert, Cologne

W. Graninger, Vienna 